\title{
Code Switching in ESL Teaching at University Level in Pakistan
}

\author{
Muhammad Younas ${ }^{1}$, Muhammad Afzaal ${ }^{2}$, Uzma Noor ${ }^{1}$, Samina Khalid ${ }^{1} \&$ Swaleha Naqvi $^{2}$ \\ ${ }^{1}$ School of Education, Soochow University, China \\ ${ }^{2}$ Department of English, Foundation University Islamabad Rawalpindi Campus, Pakistan \\ Correspondence: Muhammad Afzaal, Senior Lecturer, Department of English, Foundation University Islamabad, \\ Rawalpindi Capmus, Pakistan.
}

Received: June 20, 202

Accepted: June 29, 2020

Online Published: July 10, 2020

doi: 10.5539/elt.v13n8p63

URL: https://doi.org/10.5539/elt.v13n8p63

\begin{abstract}
Code-switching (CS) is a communicative strategy used by ESL bilingual teachers to teach English as a second language to non-native English speakers. Based on quantitative analysis of transcribed data from thirty ESL teachers at Pakistani universities, the present study examined the influence of code switching (CS) on ESL learners in Pakistan, exploring in particular the impact of CS on and teacher attitude towards CS in ESL instruction. The findings of the study indicate significant use of code-mixing and frequency of mixing words, phrase and the smallest unit of one language into another language. Furthermore, the findings suggest that CS is frequently used by the ESL teachers. The study also highlights that common nouns are followed by proper nouns, adjectives, verbs and abbreviations, with the most significant words used during instantiation of CS in the data comprising lexical items, phrases, compound words, and encouraging words. Thus, this study suggests that CS is used to facilitate the comprehension and participation of the bilingual learners.
\end{abstract}

Keywords: Education, code switching, language, ESL, Pakistan, culture

\section{Introduction}

Within the Pakistani context, English language is not only an official language but it is also the language of correspondence within governmental bodies (Hind et.al, 2020), resulting in the currency of two main dialects in Pakistan. The increasing importance of the English Language as a lingua franca, the growing dominance of the western culture, delay in making a dialect strategy and the attitude of the general public towards the English Language have influenced the greater use of code-mixing in every day interactions and communication.

In Pakistan, the official communication and correspondence are carried out in English in the majority of governmental settings. A cluster of professional fields, such as medicine, engineering, business administration, education, and information technology have adopted English as a medium of communication, According to Díaz et al. (2000), code mixing is a procedure in which lexical things and syntactic features of at least two dialects exist in a similar sentence. Many researchers such as Chugtai et.al (2016), Ahmad et.al (2015), Afzaal et.al (2020) have assayed the positive influence of CS in ESL teaching in non-native English speaking countries. Wardhaugh (1992) notes that code-switching (CS) can be defined as the sudden change of two codes or dialects without any change in the topic. Mostly, CS occurs only amongst the bilingual and multilingual speakers which is most probably related with code-switching. No change occurs in any topic while code-mixing but it occurs when speakers use multiple languages at the same time switching from one dialect or one code to another. Bilingual teachers use CS as a teaching technique to make complex topics easier for students.

Thus the main purpose of the code-switching is to facilitate bilinguals when a single language does not suffice to convey the message clearly to the reader or listener. The speakers take help of the second language to clearly convey their message and then they revert to the first language they have been speaking. Regardless of the advantages of this strategy, some changes are also observed in the message conveyed in so far as the subject-matter is concerned albeit only at sentence structure level.

Code-Mixing involves mixing:

a) Two languages

b) Two or more than two languages

c) Two or more diaglossic variations 
In addition to other types of code-switching, there is another type termed as inter-sentential code-switching. It is the first type of code switching in nature. In this particular type, the code-switching is done at sentence boundaries. This sort of code switching is often practiced between fluent and high rated language expert bilingual speakers. For instance, the Urdu text is "ME TU YAHAN HOON" but its English translation could be "I am here?" Another type of code mixing is intra-sentential code switching. In this sort of code switching, the mid of the sentence is observed as alteration, but it is not followed by any interruption or any sort of hesitation or pauses which may indicate shifting. Moreover, in this type of code-mixing speakers are not aware about the shift they have made. Considering all these, the switch is more likely to occur at the clause level including the word level. Some scholars term it as code-mixing. Poplack (cited in Romaine 1995, p.122-123) classifies code-switching as below:

(i) Tag switching

(ii) Inter-sentential switching

(iii) Intra-sentential switching

Sometimes, tags of a language are practiced in the expressions of the other languages which is understood to comprise tag-switching or extra-sentential switching. For instance, if we talk about English language, we can observe these tags, "YOU KNOW" and "I MEAN". In Urdu language, the tags can comprise "AA HA, NAA". Muysken (2000) explains that such switching mostly derives from other language for example in English, and the tag "O.K." is observed at frequency level in Urdu language speeches. The tag can occur at the midpoint of a sentence or at the end but it can also serve as a logical connector in the sentence.

Intra-sentential code-mixing instantiates within the sentence or clause or word limits with no clear change in topic. According to Poplack (1980), the third type of code-switching which is intra-sentential switching occurs when code-switching happens at a clause level or clause boundary and this also includes word boundary. An example in Urdu is the word "SHARIATIZATION" which is the adoption of Islamic laws and this word draws upon both Urdu and English. Another example is that of the English word "FAULGIRI" which is derived from word 'fault' but which is influenced by the Urdu language. This type of code-switching requires accuracy and the proficiency of the speaker in both languages. Inter-sentential code-mixing occurs when speakers who are asked to narrate a story or incident begin the narration in the L2 language and then suddenly switch back to their mother tongue. Code-switching and code-mixing can occur in all mono-lingual situations, but these two occurrences are common in bilingual and multilingual nations.

CS which occurs at sentence level or at clause level is known as inter-sentential code-switching. Poplack (1980) provides a clear example of inter-sentential code-switching by observing that when he used to start a code or message in English language "Y TERMINO EN ESPANOL" he would at times finish his sentence another language like Spanish.

Finally, the main focus of this study is on codeswitching in Urdu and Pakistani English at the levels of word, phrase, and clause and sentence. The choice of linguistic expressions depends on sociolinguistic and pragmatic factors. The difference in code-switching and code mixing is very rare or temporary because both of these types meaning Code-mixing is likewise a communicative approach. In borrowing what happens is that the linguistic features are limited to lexical units and to some extent at least have assimilations. However, code-mixing is broader as the transfer of elements is experienced at all the levels. In code-mixing, the linguistic units range from smaller level 'lexical item' to larger levels 'sentence levels'. This helps to distinguish between code-mixing and code-switching clearly (Hamers Josiane \& Blanc Michel, 1989). The most part of the examinations with respect to Code-switching gets these two terms from one single sign which is itself the code-switching. Moreover, according to these schools of thoughts, these types of code movements are very informative and knowledgeable procedures. Pfaff (1979) has differentiated these two terms as the former involves the intermixing of two languages without concerning any topic variation. Kachru (1978) also distinguishes between the two, observing that CS makes significant contributions to the effective teaching of ESL in non-native English speaking countries.

Thus code-switching means the ability to move from code one to another or language A to language B. Verbal selection in terms of roles or functions and the purpose of code-mixing are under the categories of code-switching. It is also termed linguistic exchange. This sort of exchange of linguistic units or transfer results in making a controlled or poorly controlled communication. Sometimes, code-switching is considered as a process of mixed language values or code varieties. The linguistic experts or people with multiple dialects or efficient lingual are mostly capable of using this function in various types of dialects or language mixes (Kachru 1978, p. 65). 
Other schools of thought consider these to be identical. For instance, Poplack (1980) considers inter-sentential and intra-sentential switches as code-switching, a view anticipating that of Fasold (1984) who considered these to be identical and established these as sociosemantic phenomenon with both being viewed as one strategy of coding.

At the initial stage of the development of Urdu language, the latter was enriched with Persian vocabulary. Between 1857-1947, the influence of Persian language on Urdu began to wane, and after the division of sub-continent when Pakistan gained independence, Indian Urdu built a unique relationship with the Urdu spoken in Pakistan. After independence, Urdu was adopted as the national language and locked into a relationship with English on account of the country's language policy which promoted English as an official language in Pakistan. Subsequently numerous expressions of English were added to Pakistani Urdu. There are many words like TV, glass, plate, sad, happy, thank you and sorry, good morning and good evening, dressing table and dining table, and sun, which are used in routine correspondence featuring code-mixing (Afzaal et. al. 2020). Mostly, the elite class in Pakistan makes significant use of the English language in its interactions, which has given rise to a social movement to change the monolingual society to multilingual by mixing both these codes, English and Urdu dialects.

\subsection{Status of Urdu and English Language in Pakistan}

Urdu is national language of Pakistan and it is spoken in many other countries as well, especially, in India. Significant percentage of speakers in Pakistan use Urdu language for the sake of communication (Census, 2001). In Pakistan, Urdu is the national and English is official language in Pakistan (Ethnologue, 2017; Afzaal et.al, 2019, 2020). For formal objectives, Urdu is employed predominantly even in the Punjab Province. English language is used widely in Pakistan and has been given an official-dialect status alongside Urdu. In regular Pakistani setting, it used as a corresponding dialect by the greater majority of the bilinguals. The usage of English is not quite the same as in cases wherein it is used as a first dialect. In Pakistan, as in other postcolonial nations (India, Singapore, and Nigeria and so forth.) the English dialect keeps on satisfying an extensive variety of global capacities and it used as $2^{\text {nd }}$ or $3^{\text {rd }}$ dialect in Pakistan. Indeed, Kachru (1997) has discussed the 'ideological, cultural and elitist power of English'. The majority of the general population in Pakistan are bi/multilingual. Thus English as an official language of Pakistan and global lingua franca has become the special domain as well as 'the dialect of chance, social distinction, control, accomplishment and in addition social predominance' in Pakistan.

\subsection{English-Urdu Code-Mixing in Pakistan}

English-Urdu code-mixing is a key attribute in the discourse example of the average bilingual in Pakistan. Individuals with limited information of English impress others by infusing English words into their Urdu discussion. In Pakistan, English-Urdu code-switching is used in the formal and informal discussion of educated bilingual presenters. Bilingual speakers may start the conversation with one language and switch to another language depending upon the attitude of the partner or his/her code selection. Code-switching can be noted in gatherings, lectures, courses, at home, school and college, in the workplace, at public places and so forth. Urdu is a vast language and it mixed with other languages (Kasturirangan \& Rangarajan, 2000), thus shortening the communication requirements of modern cultures as 'sudden connectivity with anybody, any time anywhere'. Thus codemixing is also evident at university level teaching. The present investigated the effect of one dialect on the other. Urdu-English code-mixing is an element of both talked and composed dialect. In the present research, an endeavor has been made to explore the phenomenon of Urdu-English code-mixing through an examination of its event in composed English talk.

Pakistani English has been discussed by many researchers (Anwar \& Talaat, 2011) in their scholarly work. These researchers have featured linguistics culturally and socially, and no study has been done yet to investigate the code mixing level in Pakistani schools. most research has looked at reasons for codemixing and as strategy but not at psychological impact of CS, Chugtai et.al., (2016) \& Ahmad et.al. (2015).

\subsection{Research Questions}

The present study was framed by the research questions below:

1. What types of code mixing are instantiated in instructional language as part of lectures at Pakistani universities?

2. What is the prevalence of CS in transcribed lecture data?

3. How does CS impact learners' classroom participation and engagement at Pakistani Universities? 
The study is substantially significant because it analyzes the instructional language of the educational system of Pakistan, a large South Asian country of geo-political significance and an emerging economy. It falls under different areas of linguistics such as sociolinguistics and stylistics. This is also important because it helps to understand the relevance and importance of codemixing in a multilingual society served by an educational system that features the use of English, Urdu and provincial languages. Thus it can serve to understand the sociolinguistic features and boundaries in the country.

\subsection{Theoretical Framework}

The linguistics analysis applied in this research is informed by Myers-Scotton's (1993) theory of Markedness Model. This model characterizes the social implications of a particular dialect decision with reference to members' commitments and is a notable model in code-switching research.

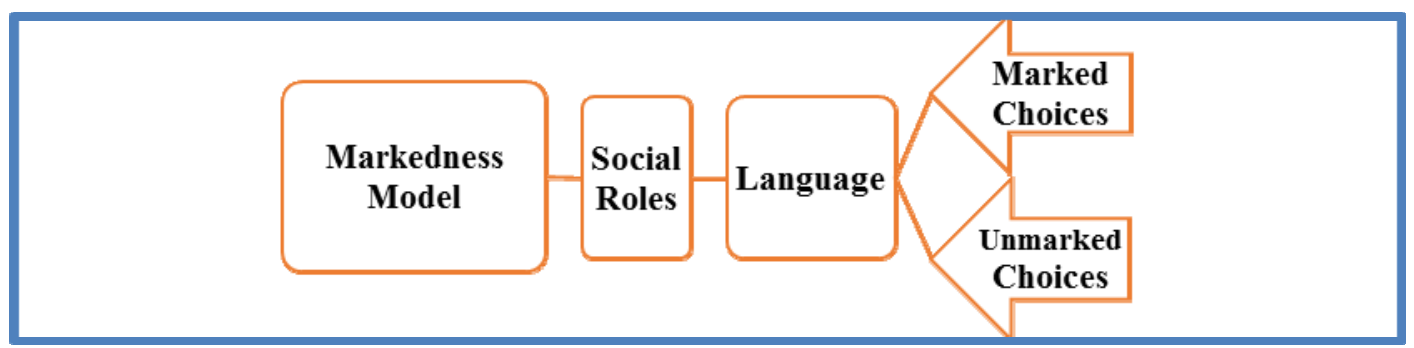

By using a specific language, the speakers indicate their understanding of a situation. It means that the 'semantic-pragmatic' decisions or 'choices' are made by the speaker according to the situation (Smith, 2002). The linguistic choice signals the specific roles of the speakers within the setting.

\section{Literature Review}

According to Muysken (2011), 'code mixing is a process in which lexical items and grammatical features of two or more languages exist in the same sentence'. Poplack (1988) defined code mixing as the 'mixing of two or more languages within a sentence', while Bhatia and Ritchie (2010 in Street \& Leung, 2010, p. 29) define it as 'the mixing of various linguistic units (morphemes, words, modifies, phrases, clauses and sentences) primarily from two participating grammatical systems within a sentence'.

As indicated by Milroy et al. (2000) individuals for the most part choose to express themselves in English because of its 'exactness' and because it requires less exertion (p. 3). Hawkins (2004) is of the view that by utilizing English words, we can 'express the most with the slightest.' He proposed that 'the (most) proficient one is the one that as the least general multifaceted nature in internet processing'. In another investigation on code-mixing by Bennui and Hashim (2014), the language utilized as a part of the Thai entertainment program was analyzed. Information was recorded from well-known entertainment programs. The outcome demonstrated that seven sorts of code mixing were used as a part of the program, and that participants were 'mixing the two codes that are Thai and English dialect, clipping, transliteration truncation, hybridization, English names and others'. Leung (2010) observes that 'code mixing has moved toward becoming socially and openly unavoidable' in the current globalized society in which individuals from various dialects and societies can interface or convey thoughts through advancements.

Early studies on this area were limited to specific domains of academic discourse. One study looked at the number of English words used in the Urdu conversation within composed data from 200 BS students of 5 universities which are located in Lahore, Pakistan (Mann \& Stewart, 2000). According to Khan (2014) these components incorporate sexual orientation, geographical background, financial class, and education. Saleemi (2003) highlighted that 'require a decent arrangement of socio-social contribution to get a solid shape' which implies that the dialect we talk in and the importance is surmised from society in which we live and the way of life which we take after. For Talaat (2005), code-mixing in discourse is not just a linguistic phenomenon but also represents an important social capacity of English in Pakistan which signals exceptional linguistic change. In another study (Dar, Akhtar et al. 2014), data analysis showed that code-mixing is a crucial part of Urdu news. It was noticeable that exclusive external code mixing happened in the recorded news. Alam (2006) observed the frequency of code mixing in the language of Bangladesh speakers and found that the English occasionally helped people to draw the attention of others. Aslam et al (2011) point out that British peoples imposed their civilization, culture, behaviors especially their language during colonial period in subcontinent. Ranjha (2012) notes that Urdu language has the capacity for integrating many words from other languages in vocabulary and that in Pakistan many people belonging to different fields use English words in casual social interaction and gossip. 
Rasul (2006) notes that a society is interlinked with language, media and culture. Mushtaq and Zahra (2012) have analyzed code mixing in TV ads. The aim of their research was to analyse the degree and effect of code-mixing in promotions appearing on different Pakistani TV channels.

Reppen (1994) conducted research on school data of elementary level. Data collected from a junior standard grade to highlight different dimensions. The same research was repeated on the large scale to mention and confirm the data (Donley \& Reppen, 2001). Code- mixing has moved toward becoming socially and openly unavoidable in the current globalized society in which individuals from various dialects and societies can interface or convey thoughts through advancements. Rasul (2013) states that code-mixing occurs when vocabulary and grammar of both languages act in same sentence. He further adds that when two speaker talks with each other by using more than one codes or dialects, they create half and half another variety and kind of the dialect. As indicated by Wardhaugh (1992) code mixing happens when speakers use two dialects together to demonstrate that they change from one language to others in one expression.

The mutual intelligibility and its level are measured in the form of percentage and it is a ratio in the language for the understandable units. It is irregular or uneven which may be explained as people from one language can also understand other languages as well. Simpson (2007) defined mutual intelligibility in his research as an interchangeable connection between codes or dialects. It occurs amongst the languages or codes. We can have many examples of mutual intelligibility between different languages such as Afrikaans and Dutch languages or Slovak and Czech, and many other languages as well. Moreover, some languages like Ukrainian and Polish languages are examples and sources of mutual intelligible languages but in spoken form and other languages such as Faroese and Icelandic are only in written form (Siegel, Lynch \& Eades, 2007).

In his research, Asghar (2012) found code-mixing to be hybridized, adding that irrespective of the value of these two dialects or codes, a new dialect and code with a new vocabulary and punctuation marks tend to evolve. This sort of new dialect or code carries its own type of mixed dialect. Asghar provides a number of examples for such code-mixing, for instance class on, machine, leader, hakomati-memberan and quomi-assembly. In addition, this, he adds that sometimes people try to show off their status through the use of English language. In a study titled 'The Form and Functions of English in Pakistan', Talaat (2002) gathered data from English daily newspapers and magazine book in Pakistan, examining and comparing examples in these with British English of good standard with the help of Urdu language sentence structures.

\section{Research Methodology}

The aim of this research was to investigate English code mixing deployed in teaching at university level in Pakistan. In line with earlier studies (for instance, Nadeem (2012), qualitative and quantitative techniques were deployed in the present study to explore codemixing in the research setting. Dörnyei (2007) has also supported the use of quantitative, qualitative and mixed methods of research in the field of applied linguistics.

A total of thirty lectures were attended and transcribed to identify English code mixing during delivery of different lectures in three different universities in Pakistan. In this way, data were investigated minutely because the analysis of collecting data was aimed at determining the interactive elements that provide great knowledge about the actual of second language (English) in the native language (Urdu).

\subsection{Population \& Sampling}

The study took participants from the University of Punjab, University of Education and BZU as the population of this research. A total of thirty classes of BS level from the department of Education were attended by the researcher and all lectures were transcribed to know the frequency of English during teaching in Urdu Language. Ten lectures from each university were selected as sample from the whole population and the time-frame of the populated data comprised the BS in Education class session 2016-2020. These lectures were selected simultaneously to obtain comparable results.

\subsection{Transcription of Data}

Code mixing is a dominant strategy used by the teachers of the Education departments at these three universities to deliver lectures to BS classes. To obtain the code-mixing frequency of English in Urdu lectures, all thirty lectures were transcribed to check the code-mixing frequency. The aim was to identify the most frequent English words used by teachers of Education departments at the selected universities. The frequency of the occurrence of these words was identified and tabulated. English words were noted at individual level, phrase level, compound level and abbreviations. The results have been presented in the form of figures and table. 


\subsection{Urdu English Code-Mixing Explanation}

As Urdu is the national language of Pakistan which is embedded in culture, traditional values, Urdu words cannot be avoided or replaced with English words easily. Thus there was prevalent use of Urdu figurative language which deals with the pure and local cultural and social realities in Pakistan to serve the communication needs within the research context. Regardless of the figurative linguistic values of Urdu, the researcher encountered some limitations in Urdu language. During transcribing the teacher's lectures, the researchers have seen the use of English figurative strategies such as similes, metaphors, and idioms in Urdu discussion. English metaphors plays important role in evaluative functions. The process of meaning adaptation of changed English words are very common in spoken Urdu discourse. Those English words have a habit of to develop semantic postponements by obtaining new senses or new shades of meaning.

\section{Results and Discussion}

Analysis of data showed that code-mixing plays a pivotal role in Pakistani education system. Exclusive external code mixing was observed in selected lectures. Code mixing was identifiable at word level for the most part and at phrase level to a lesser extent.

Frequency of Code-mixing

\begin{tabular}{cccccc}
\hline Sr. No & $\begin{array}{c}\text { Total Number of } \\
\text { Words }\end{array}$ & Single Lexical Items & $\begin{array}{c}\text { Compound } \\
\text { Words }\end{array}$ & Phrases & $\begin{array}{c}\text { Abbreviati } \\
\text { ons }\end{array}$ \\
\hline $\begin{array}{c}\text { University of } \\
\text { Punjab }\end{array}$ & 13096 & 1239 & 386 & 207 & 109 \\
$\begin{array}{c}\text { University of } \\
\text { Education }\end{array}$ & 9705 & 1133 & 373 & 195 & 158 \\
BZU & 13283 & 1425 & 419 & 197 & 100 \\
\hline Total & $\mathbf{3 6 0 8 4}$ & $\mathbf{3 7 9 7}$ & $\mathbf{1 1 7 8}$ & $\mathbf{5 9 9}$ & $\mathbf{3 6 7}$ \\
\hline
\end{tabular}

The total number of words were counted in scripted thirty lectures. These totaled 36084 words across all 30 transcribed lectures. 13096 words were used during ten lectures in University of Punjab, 9705 words were used in University of Education and 13283 words were used in the dataset from BZU. Frequency analysis also shows that the 3797 English words used comprised single lexical words, whereas 1178 English words were used as compounds words, and 599 words were English phrases. Further, 367 English abbreviations were used in the complete dataset.

University of Punjab Lecture Detail

\begin{tabular}{cccccc}
\hline Sr. No & Total Number of Words & Single Lexical Items & Compound Words & Phrases & Abbreviations \\
\hline 1 & 1345 & 143 & 29 & 15 & 12 \\
2 & 1534 & 200 & 41 & 20 & 4 \\
3 & 1734 & 149 & 37 & 29 & 10 \\
4 & 1400 & 135 & 40 & 23 & 14 \\
5 & 1604 & 155 & 54 & 19 & 9 \\
6 & 1339 & 120 & 49 & 30 & 13 \\
7 & 1218 & 95 & 60 & 27 & 19 \\
8 & 800 & 85 & 17 & 8 & 7 \\
9 & 1330 & 109 & 30 & 27 & 9 \\
10 & 792 & 48 & 29 & 9 & 5 \\
\hline Total Number & $\mathbf{1 3 0 9 6}$ & $\mathbf{1 2 3 9}$ & $\mathbf{3 8 6}$ & $\mathbf{2 0 7}$ & $\mathbf{1 0 2}$ \\
\hline Percentage & & $\mathbf{9 . 4 6 \%}$ & $\mathbf{2 . 9 4 \%}$ & $\mathbf{1 . 5 8 \%}$ & $\mathbf{0 . 7 7 \%}$ \\
\hline
\end{tabular}


Descriptive Statistics from Punjab University lecture dataset

\begin{tabular}{ccccc}
\hline & $\begin{array}{c}\text { Total } \\
\text { Shows }\end{array}$ & Mean & Variance & Std. Deviation \\
\hline Total Number of words & 10 & 2484.7 & 13997023.57 & 3741.259623 \\
Single lexical Items & 10 & 233.5 & 126576.0556 & 355.775288 \\
Compound words & 10 & 74.3 & 12154.23333 & 110.2462395 \\
Phrases & 10 & 39.9 & 3506.989 & 59.21984 \\
Abbreviations & 10 & 19.2 & 866.1777778 & 29.43089835 \\
\hline
\end{tabular}

Analysis of data shows that a total 13096 words used in ten selected lectures. The single lexical English words 1239 comprised $9.46 \%$ of all words in all lectures. The compound English words totaled 386, comprising 2.94\% of 13096 words of all 10 transcribed lectures. Also, 207 English phrases were used representing $1.58 \%$ of all words used and 102 abbreviations are used representing .77\% of all words. In descriptive statistics, some important statistical tests also show values of all five dimensions means totaling words statistics, lexical words, compound words, phrases and abbreviations. The statistical values of these items are clearly mentioned in the descriptive statistical table presents Means, Variance and Std. Deviations of all. These statistics clearly show the extent to which code-mixing is used in these lectures.

University of Education's lectures detail

\begin{tabular}{cccccc}
\hline Sr. No & $\begin{array}{c}\text { Total Number of } \\
\text { Words }\end{array}$ & $\begin{array}{c}\text { Single Lexical } \\
\text { Items }\end{array}$ & $\begin{array}{c}\text { Compound } \\
\text { Words }\end{array}$ & Phrases & $\begin{array}{c}\text { Abbreviatio } \\
\text { n }\end{array}$ \\
\hline 1 & 899 & 114 & 29 & 18 & 8 \\
2 & 1108 & 132 & 37 & 21 & 5 \\
3 & 778 & 105 & 41 & 25 & 11 \\
4 & 949 & 122 & 50 & 19 & 17 \\
5 & 894 & 117 & 29 & 15 & 21 \\
6 & 1243 & 144 & 51 & 27 & 14 \\
7 & 923 & 108 & 43 & 20 & 32 \\
8 & 787 & 89 & 19 & 11 & 17 \\
9 & 1205 & 137 & 41 & 25 & 18 \\
10 & 919 & 65 & 33 & 14 & 15 \\
\hline Total Number & $\mathbf{9 7 0 5}$ & $\mathbf{1 1 3 3}$ & $\mathbf{3 7 3}$ & $\mathbf{1 9 5}$ & $\mathbf{1 5 8}$ \\
\hline Percentage & & $\mathbf{1 1 . 6 7 \%}$ & $\mathbf{3 . 8 4} \%$ & $\mathbf{2 . 0 0 \%}$ & $\mathbf{1 . 6 2 \%}$ \\
\hline
\end{tabular}

Descriptive Statistics of dataset from Education University

\begin{tabular}{ccccc}
\hline & $\begin{array}{c}\text { Total } \\
\text { Shows }\end{array}$ & Mean & Variance & Std. Deviation \\
\hline Total Number of words & 10 & 970.5 & 26135.16667 & 161.6637457 \\
Single lexical Items & 10 & 113.3 & 551.5666667 & 23.48545649 \\
Compound words & 10 & 37.3 & 100.0111111 & 10.00055554 \\
Phrases & 10 & 19.5 & 27.16667 & 5.212165 \\
Abbreviations & 10 & 15.8 & 55.73333333 & 7.465476096 \\
\hline
\end{tabular}

The transcribed data of Education University shows that 9705 words were used in 10 selected lectures. 1133 words were used as single English lexical words, comprising 11.67\% of ten lectures. Compound English words numbered 373 which made up 3.84\% of the dataset. Similarly, 195 words used as English phrases were 2\% of the dataset. Abbreviations used in these lectures totaled 158 words which made up $1.62 \%$ of the dataset. The descriptive statistics clearly show the code mixing level used in these lectures. 
BZU lectures detail

\begin{tabular}{cccccc}
\hline Sr. No & Total Number of Words & Single Lexical Items & Compound Words & Phrases & Abbreviations \\
\hline 1 & 1145 & 155 & 35 & 12 & 6 \\
2 & 1886 & 212 & 48 & 25 & 2 \\
3 & 1903 & 178 & 45 & 34 & 9 \\
4 & 1876 & 148 & 57 & 28 & 38 \\
5 & 1644 & 177 & 64 & 16 & 6 \\
6 & 1299 & 137 & 45 & 25 & 17 \\
7 & 1417 & 168 & 56 & 24 & 5 \\
8 & 577 & 80 & 11 & 10 & 11 \\
9 & 1038 & 111 & 37 & 20 & 2 \\
10 & 498 & 59 & 21 & 3 & 4 \\
\hline Total Number & $\mathbf{1 3 2 8 3}$ & $\mathbf{1 4 2 5}$ & $\mathbf{4 1 9}$ & $\mathbf{1 9 7}$ & $\mathbf{1 0 0}$ \\
\hline Percentage & & $\mathbf{1 0 . 7 2 \%}$ & $\mathbf{3 . 1 5 \%}$ & $\mathbf{1 . 4 8 \%}$ & $\mathbf{0 . 7 5 \%}$ \\
\hline Descriptive Statistics of BZU & & & & \multicolumn{2}{c}{ Std. Deviation } \\
\hline \multicolumn{2}{c}{ Total Number of words } & 10 & 1328.3 & 269044.4556 & 518.6949542 \\
Single lexical Items & 10 & 142.5 & 2224.277778 & 47.1622495 \\
Compound words & 10 & 41.9 & 270.5444444 & 16.4482353 \\
Phrases & Total & 10 & 19.7 & 88.23333 & 9.39326 \\
Abbreviations & 10 & 10 & 117.3333333 & 10.83205121
\end{tabular}

The analysis shows the number of words in these transcribed lectures totaled 13283. Teachers used 1425 English words as single lexical words which comprised $10.72 \%$ of all words. 419 words were also used as compound English words comprising 3.15\% of the total words. 197 phrases were used as English phrases which $1.48 \%$. Likewise, 100 abbreviations are used in these 10 lectures which comprise $75 \%$ of 13283 words. The descriptive statistic table also shows the statistical values of code-mixing in these lectures.

\subsection{Box Plots Description}

After quantitative analysis of the data, comprehensive box plots for clear-cut description and presentation of all three datasets were designed. In these box plots, four dimensions are discussed as the researcher presented has focused on these dimensions in all three groups. The dimensions comprise single lexical words, compound words, phrases and abbreviations.

Explanatory Table

\begin{tabular}{ccc}
\hline S.N & TYPES & EXAMPLE \\
\hline 1 & Single Lexical words & Meeting, letter, nice, clear, punish. \\
2 & Compound words & Supreme court, one-sided, status grant. \\
3 & Phrases & Head of appointment, as a guest, as an analyst. \\
4 & Abbreviations & PSO, DG, IMF, \\
\hline
\end{tabular}

The researcher used statistical software obtaining the results. The comparative analysis of all four dimensions is presented in the box plots. 


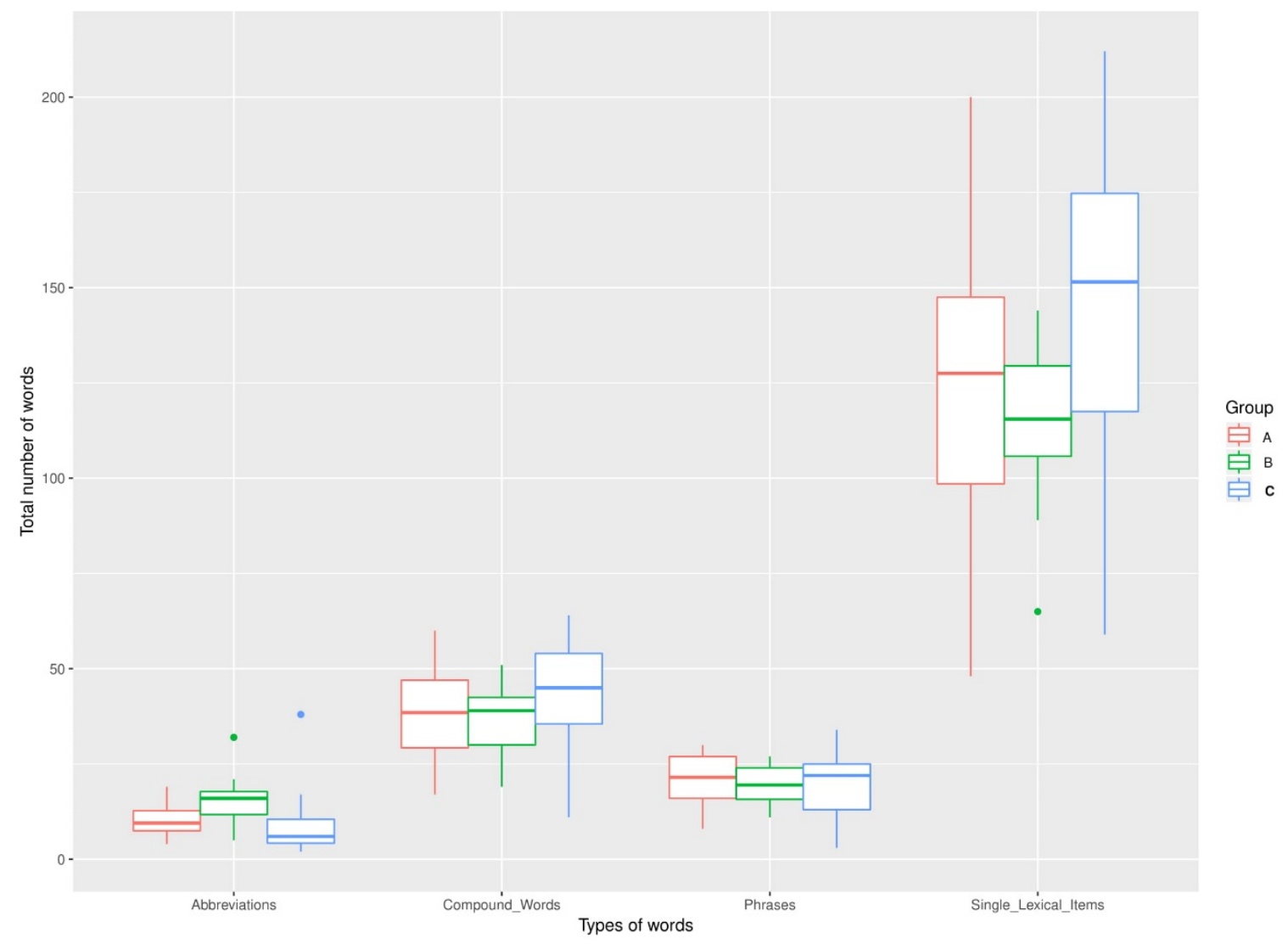

The analysis of thirty lectures showed that code mixing was frequently deployed by university teachers while delivering lectures to students. Although the English words have their own equivalent in Urdu language which tended to be used in the past, with the change in social attitude, the English words are used directly in Urdu language rather than being translated, for example words like a break and welcome back. Furthermore, it was notable that even those English words the equivalents of which are known are adopted in daily life conversations. The box plot shows that single lexical items are counted the maximum number of times in this code-mixed data. The teacher and student turns were also analyzed to identify how CS impacted student participation and interaction. This analysis showed that teacher participants tended to use CS for extending explanations, offering clarifications, inviting questions, showing solidarity with the learners and carrying out comprehension checks. These functions of CS facilitated learner engagement and participation and allowed for the smooth progress of the lecture and interaction.

\section{Conclusion}

The paper concludes that flexibility in terms of code-switching ensures the successful transfer of knowledge. Teachers should practice CS in classroom as a tool which enables the learners comprehend the technical and ambiguous sections of ESL lesson. In addition, it promotes a positive outcome on the part of learners and creates a friendly environment in classroom. The study sums up the purpose of CS as a substantive tool for bilingual and multicultural classes. Teachers in the Pakistani universities tend to rely heavily upon the use of English words in offering code-mixed instructions, clarifications, explanations and encouragement to students. Therefore, there is a need to research this phenomenon further.

\subsection{Recommendations}

Based on analysis of the data, it is evident that CS is predominantly used by teachers in their lectures in order to progress content delivery and to negotiate teacher and student interactions. However, it is important for university teachers in ESL contexts like Pakistan to develop a theorized understanding of CS, its roles and functions and impact upon student learning. In this regard, teacher trainings should be offered to the teachers to demonstrate the effective use of CS as a pedagogical strategy. Using CS intuitively, rather than with a clear understanding of its theorized basis is likely to limit the transfer of its benefits to learners and also lead to a great deal of variation in how it is implemented as a pedagogical strategy. If teachers are trained in the use of CS, they 
can leverage student teacher interactions during the course of the lecture more effectively, attaining the outcomes they want in a precise manner linked to instructional objectives.

University management also needs more awareness as to the efficacy of CS as a pedagogical strategy. At present, with English being the official medium of instruction at tertiary institutions in Pakistan, senior management and academic managers tend to view predominant use of CS as a drawback rather than pedagogical advantage for learners. Awareness raising and trainings can help to address this issue, thus enabling management to support the judicious use of CS as a pedagogical strategy.

Given the prevalence of codemixing in Pakistani educational institutions, it is important to explore not just the type of codemixing that transpires but also what functions it serves. Future research may look at other variables of influence not considered in this study due to the restrictions of scope, such as the influence of teacher and student genders on the deployment of codemixing or of the influence of disciplinary domains on the use (or not) of codemixing in Pakistani academia. Researchers may also consider building upon this study by interviewing teachers and students as to what functions codemixing serves in their teaching and learning context.

\section{References}

Abbas, F., et al. (2011). Code-Mixing as a Communicative Strategy among the University Level Students in Pakistan. Language in India, 11(1).

Alam, S. (2006). Code-Mixing in Bangladesh: A Case Study of Non-Government White-Collarservice Holders and Professionals. Asian Affairs, 28(4), 52-70.

Alfadda et.al. (2020). A Study of Needs Analysis of Education at Undergraduate Level: EFL Case Study. International Journal of Psychosocial Rehabilitation, 24(8).

Anwar, B. \& M. Talaat. (2011). English in non-native context: Distinctive features of Pakistani journalistic English. English Language and Literary Forum. Annual Research Journal.

Afzaal, M., et.al. (2020). A Pragmatics analysis of Michael Scott and the Violation of Conversational Maxims in The Office Television Series. International Journal of Disaster Recovery and Business Continuity. 11(1), 2217-2229.

Afzaal, M., Khan, M., Ghaffar Bhatti, A., \& Shahzadi, A. (2019). Discourse and Corpus-based Analysis of Doctor-Patient Conversation in the Context of Pakistani Hospitals. European Online Journal of Natural and Social Sciences, 8(4), 732-752.

Ahmad, R. I., Ahmed, R. S., Rauf, M. A., \& Seemab, G. M. (2015). Concept of the Code Switching and Code Mixing as Facilitators in Urdu EFL Class. Journal of Policy Research (JPR), 1(2), 62-66.

Afzaal, M., Liu, K., Wu, B., Sayyida, R., \& Naqvi, S. B. (2020). An Investigation of Abstract and Discussion Sections in Master's Dissertations. International Journal of English Linguistics, 10(1). https://doi.org/10.5539/ijel.v10n1p220

Chughtai, I. A., Khan, M. A., \& Khan, M. R. (2016). Reasons and contexts to switch and mix English code by Pakistani young learners in their native speech: A sociolinguistic study. International Journal of Language and Linguistics, 3(1), 85-94.

Dar, M. F., et al. (2014). Code-switching in Pakistani English language classrooms: perceptions of English language teachers. Journal of Social Sciences and Interdisciplinary Research, 2(2), 16-26.

Donley, K. K. \& R. Reppen. (2001). Using corpus tools to highlight academic vocabulary in SCLT. TESOL Journal, 10(2-3), 7-12.

Dörnyei, Z. (2007). Research methods in applied linguistics: Quantitative, qualitative, and mixed methodologies, Oxford University Press.

Kachru, B. B. (1997). World Englishes and English-using communities. Annual review of applied linguistics. 17, 66-87. https://doi.org/10.1017/S0267190500003287

Kasturirangan, K. \& S. Rangarajan. (2000). 4. Satellite Communications and its Impact on Society. Emerging Communication Technologies and the Society, 43.

Khan, A. M. (2014). Social aspects of code-switching: An analysis of Pakistani television advertisements. Information Management and Business Review, 6(6), 269. https://doi.org/10.22610/imbr.v6i6.1125

Mann, C. \& F. Stewart. (2000). Internet communication and qualitative research: A handbook for researching online, Sage. https://doi.org/10.4135/9781849209281 
Mushtaq, H. and T. Zahra. (2012). An Analysis of Code-Mixing in Television Commercials. Language in India, 12(11).

Muysken, P., et al. (2000). Bilingual speech: A typology of code-mixing, Cambridge University Press.

Myers-Scotton, C. (1993). Common and uncommon ground: Social and structural factors in codeswitching. Language in society, 22(4), 475-503. https://doi.org/10.1017/S0047404500017449

Nadeem, M. (2012). Urlish: A code switching/code mixing pedagogical approach in teacher education. JRRE, $6(2)$.

Ranjha, M. I. (2012). Urdu Syllable: Templates and Constraints. LANGUAGE \& TECHNOLOGY, 29.

RASUL, S. (2006). Language hybridization in Pakistan as socio-cultural phenomenon: An analysis of code-mixed linguistic patterns, National University of Modern Languages Islamabad.

Rasul, S. (2013). Borrowing and Code Mixing in Pakistani Children Magazines: Practices and Functions. Pakistan: A journal of Pakistan studies, 5(2), 46-72.

Reppen, R. (1994). A genre-based approach to content writing instruction. TESOL Journal, 4(2), 32-35.

Saleemi, A. P. (2003). Mental and social aspects of Linguistic reality: The mind's language and its social reality. Pakistani Languages and Societies: Problems and Prospects, 115-120.

Smith, D. J. (2002). Patterns of variation in Spanish/English bilingualism in northeast Georgia.

Talaat, M. (2005). Convergence and Social Context in Pakistani English. ELF Annual Research Journal, 4, $27-48$.

Yiamkamnuan, J. (2010). The implication of English-Thai mixing and possibility of internet chat rooms as alternative learning environments. Retrieved October 10, 2013.

\section{Copyrights}

Copyright for this article is retained by the author(s), with first publication rights granted to the journal.

This is an open-access article distributed under the terms and conditions of the Creative Commons Attribution license (http://creativecommons.org/licenses/by/3.0/). 